\title{
Robust Adaptive Fuzzy Output Tracking Control for a Class of Twin-Roll Strip Casting Systems
}

\author{
Yu-Jun Zhang, ${ }^{1}$ Li-Bing Wu, ${ }^{2}$ Hong-Yang Zhao, ${ }^{3}$ Xiao-Dong $\mathrm{Hu}^{3}$ \\ Wen-Yu Zhang, ${ }^{4}$ and Dong-Ying $\mathrm{Ju}^{3}$ \\ ${ }^{1}$ Software School, University of Science and Technology Liaoning, Anshan, Liaoning 114051, China \\ ${ }^{2}$ School of Science, University of Science and Technology Liaoning, Anshan, Liaoning 114051, China \\ ${ }^{3}$ School of Materials and Metallurgy, University of Science and Technology Liaoning, Anshan, Liaoning 114051, China \\ ${ }^{4}$ College of International Finance and Bank, University of Science and Technology Liaoning, Anshan, Liaoning 114051, China
}

Correspondence should be addressed to Dong-Ying Ju; dyju_lkd@163.com

Received 2 January 2017; Revised 22 March 2017; Accepted 11 April 2017; Published 5 June 2017

Academic Editor: Xuejun Xie

Copyright (C) 2017 Yu-Jun Zhang et al. This is an open access article distributed under the Creative Commons Attribution License, which permits unrestricted use, distribution, and reproduction in any medium, provided the original work is properly cited.

\begin{abstract}
This paper is concerned with the adaptive fuzzy control problem for a class of twin-roll strip casting systems. By using fuzzy logic systems (FLSs) to approximate the compounded nonlinear functions, a novel robust output tracking controller with adaptation laws is designed based on the high gain observer. First, the nonlinear dynamic equations for the roll gap and the molten steel level are constructed, respectively. Then, the mean value theorem is employed to transform the nonaffine nonlinear systems to the corresponding affine nonlinear systems. Moreover, it is also proved that all the closed-loop signals are bounded and the systems output tracking errors can converge to the desired neighborhoods of the origin via the Lyapunov stability analysis. Finally, simulation results, based on semiexperimental system dynamic model and parameters, are worked out to show the effectiveness of the proposed adaptive fuzzy design method.
\end{abstract}

\section{Introduction}

As is well known, the strip casting combines two processes of continuous casting and hot rolling into a single production; consequently, it brings in a lot of advantages including lower investment cost, energy saving, less space requirements. More specifically, compared with the conventional continuous casting [1], the production line and the production cost of the twin-roll strip casting process are significantly shortened and reduced, respectively. Meanwhile, due to a high cooling rate for the strip casting, the mechanical properties of metallic materials can be increased [2]. Nevertheless, the strip casting process is always with nonlinear uncertainty, external disturbance, and coupled behaviors, and the roll gap and the molten steel level control problems are still important research topics to guarantee steel strip quality.

In [3], the model of the continuous casting process with various nonlinearities was proposed, and the corresponding controller was also designed. The authors in [4] developed an adaptive algorithm for the mould level control of a continuous steel slab casting. The modeling and control problem for a class of twin-roll strip casting system was studied in [5]. Correspondingly, some successful adaptive fuzzy or neural network approaches for the molten steel level control have been studied (see, e.g., [6-8] and the references therein) in the casting process. Recently, based on the perturbation method, a decoupling control strategy in [9] was proposed to obtain a uniform sheet thickness and keep a constant roll separating force in the strip casting process. By using twin-roll casting technology (TRC), the optimized process parameters and their effects on TRC of 7050 aluminum alloys strips were studied in [10].

It should be pointed out that, in most of the results about nonlinear systems, the considered parameter uncertainties and disturbances satisfy matching condition [11, 12]. Besides, the above-mentioned control approaches require that all the states of the systems are available; thus they cannot be applied to nonaffine nonlinear system with unmeasured states. In 
particular, for the roll gap and the molten steel level control of twin-roll strip casting process, it is difficult to measure the rates of change of the roll gap and the molten steel level by using proper sensors. In [13], the adaptive fuzzy tracking control problem for a class of uncertain nonaffine nonlinear systems with nonsymmetric dead-zone inputs was investigated; however, the proposed approaches can only handle the SISO nonaffine nonlinear systems rather than MIMO systems with complex coupling terms. To the best knowledge of the authors, it is the first time the adaptive fuzzy tracking control is developed for MIMO nonaffine nonlinear casting systems with immeasurable states, which are very meaningful and more practical.

The above considerations motivate our study work. Especially, inspired by $[8,13]$, by means of fuzzy approximation technique, the adaptive fuzzy output tracking control problem for a class of twin-roll strip casting systems is considered. Moreover, compared with the existing results, the main contributions of this paper are as follows: (1) The novel fuzzy tracking controllers with adaptation laws are designed by using fuzzy logic systems to approximate the compound nonlinear functions. (2) In order to handle the nonaffine coupling terms, the implicit function theorem and the mean value theorem are invoked, respectively. It is thus that the MIMO nonaffine nonlinear system can be transformed into the corresponding affine nonlinear system by this way. (3) By making use of adaptive mechanism driven by the estimation states obtained from the high gain observer, the influence of nonlinear parameter uncertainties and external disturbances is restrained effectively. It is also shown that the output tracking errors of the roll gap and the molten steel level can converge to the desired neighborhoods via the Lyapunov stability analysis.

The rest of the paper is organized as follows. In Section 2, the mathematical model for the strip casting process is given. The adaptive fuzzy output tracking control problem is addressed in Section 3. Simulations and experimental analysis are then provided in Section 4 to verify the effectiveness of the proposed approach. Finally, Section 5 draws the conclusions.

\section{System Model for the Strip Casting Process}

2.1. Molten Metal Level Equation. In this subsection, a diagram of the strip casting process is shown in Figure 1, and the corresponding mathematical model for the molten steel leveling dynamics is described as in [8]. Concretely, the following dynamic equation can be derived from (1)-(4) in [8]

$$
\frac{d x_{h}}{d t}=F^{-1}\left(x_{g}, x_{h}\right) L^{-1}\left(Q_{\text {in }}-Q_{\text {out }}-L x_{h} \frac{d x_{g}}{d t}\right),
$$

where $R$ is the roll radius, $L$ is the length of the roll cylinders, $Q_{\text {in }}$ and $Q_{\text {out }}$ are the input flow and output flow of the pool between the two rolls, respectively, and $F\left(x_{g}, x_{h}\right)=x_{g}+2 R-$ $2 \sqrt{R^{2}-x_{h}^{2}}$.

In addition, for convenience, the input flow $Q_{\text {in }}$ is taken as $Q_{\text {in }}=k(t) u$, where $u$ is the control input, and the gain $k(t)$ is

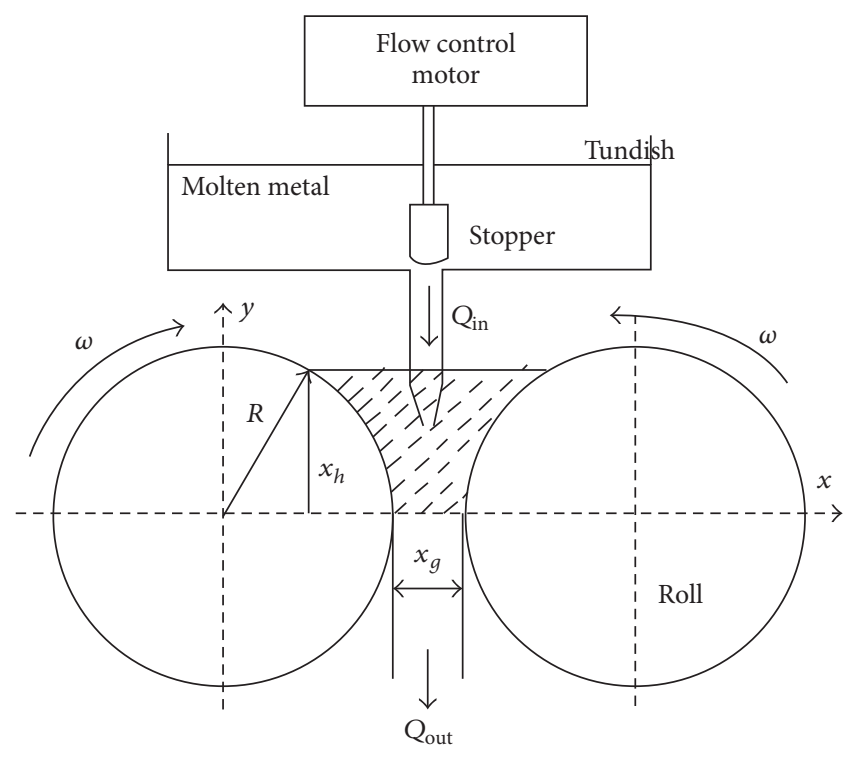

FIGURE 1: Schematic diagram of the twin-roll casting process.

determined empirically. The output flow $Q_{\text {out }}$ can be derived from the product of roll surface tangential velocity $v$, roll gap $x_{g}$, and the length of the roll cylinder $L$; that is, $Q_{\text {out }}=L x_{g} v$. So the molten metal level equation (1) is rewritten as

$$
\frac{d x_{h}}{d t}=F^{-1}\left(x_{g}, x_{h}\right) L^{-1}\left(k u-L x_{g} v-L x_{h} \frac{d x_{g}}{d t}\right) .
$$

By introducing the coordinate transformations $x_{11}=x_{g}$, $x_{12}=d x_{g} / d t, x_{21}=x_{h}$, and $x_{22}=d x_{h} / d t$, it follows from (2) that the following MIMO nonaffine nonlinear system can be obtained:

$$
\begin{aligned}
\dot{x}_{i 1} & =x_{i 2} \\
\dot{x}_{i 2} & =f_{i}\left(x, u_{i}\right)+d_{i} \\
y_{i} & =x_{i 1}, \quad i=1,2,
\end{aligned}
$$

where $x=\left[x_{1}, x_{2}\right]^{T}$ and $x_{i}=\left[x_{i 1}, x_{i 2}\right]^{T}$ stand for the state variables, $d_{i}$ is the unknown disturbance, $y_{i}$ and $u_{i}$ denote the system output and the control input of the $i$ th subsystem, respectively, and $f_{i}\left(x, u_{i}\right)$ is the unknown and smooth nonaffine nonlinear function with $u_{i}$ being electric servomotor control.

Remark 1. Applying Newton's Second Law, it is easy to obtain that $f_{1}\left(x, u_{1}\right)=(1 / M)\left(K u_{1}-f_{M}(x)\right)$ with $M$ being the mass of roll, $f_{M}(x)$ being viscous resistance, and $u_{1}$ being servomotor control. At the same time, the nonaffine nonlinear function $f_{2}\left(x, u_{2}\right)$ can be derived by taking derivative of (2).

The objective of this paper is to design an adaptive fuzzy output feedback controller $u_{i}$ such that all the closed-loop error signals are uniformly ultimately bounded, and the system output $y_{i}$ tracks a reference trajectory $y_{d i}$ within a desired compact set in the presence of unknown nonaffine 
nonlinear coupling term. Then, to ensure the feasibility of the considered problem, the necessary assumptions are required for the nonaffine nonlinear system (3).

Assumption 2. The desired reference trajectory $y_{d i}$ is known and smooth, and its derivative is also continuous. That is, there exist unknown positive constants $\bar{d}_{i}, \overline{\dot{d}}_{i}$, and $\overline{\ddot{d}}_{i}$ such that $\left|y_{d i}(t)\right| \leq \bar{d}_{i},\left|\dot{y}_{d i}(t)\right| \leq \overline{\dot{d}}_{i}$, and $\left|\ddot{y}_{d i}(t)\right| \leq \overline{\vec{d}}_{i}$, respectively.

Assumption 3 (see [13]). For all $x \in \mathbb{R}^{4}$ and $u_{i} \in \mathbb{R}$ in the $i$ th subsystem (3), there always exist positive constants $f_{i 1}$ and $f_{i 2}$ such that the following inequality holds:

$$
0<f_{i 1} \leq \frac{\partial f_{i}\left(x, u_{i}\right)}{\partial u_{i}} \leq f_{i 2}, \quad i=1,2 .
$$

Assumption 4. The external disturbance $d_{i}$ is bounded; that is, there exists an unknown positive constant $d_{i}^{*}$ such that $\left|d_{i}(t)\right| \leq d_{i}^{*}$.

Remark 5. It can be seen that Assumptions 2 and 4 are quite standard in most of the references for nonlinear tracking control, which means that the external disturbances, the reference signals, and their derivatives are bounded. Assumption 3 is used to decouple the nonaffine nonlinear term for the $i$ th subsystem, which implies that the change rate of the control input gain is bounded.

2.2. Fuzzy Logic Systems (FLSs). Generally speaking, for an FLS, it consists of four parts: the knowledge base, the singleton fuzzifier, product inference, and center average defuzzifier, respectively. First, construct the knowledge base for FLS with the following IF-THEN rules:

$$
R_{i} \text { : If } X_{1} \text { is } F_{1}^{i} \text { and } \ldots \text { and } X_{n} \text { is } F_{n}^{i} \text {. }
$$$$
\text { Then } Y \text { is } B_{i}, i=1,2, \ldots, N \text {. }
$$

Next, the FLS with the singleton fuzzifier, product inference, and center average defuzzifier can be expressed as

$$
Y(X)=\frac{\sum_{i=1}^{N} \theta_{i} \prod_{j=1}^{n} \mu_{F_{j}^{i}}\left(X_{j}\right)}{\sum_{i=1}^{N}\left[\prod_{j=1}^{n} \mu_{F_{j}^{i}}\left(X_{j}\right)\right]},
$$

where $X=\left[X_{1}, X_{2}, \ldots, X_{n}\right]^{T} \in \mathbb{R}^{n}, \mu_{F_{j}^{i}}$ is the membership of $F_{j}^{i}$, and $\theta_{i}=\max _{Y \in \mathbb{R}}\left[\mu_{B_{i}}(Y)\right]$. Let

$$
\phi_{i}(X)=\frac{\prod_{j=1}^{n} \mu_{F_{j}^{i}}\left(X_{j}\right)}{\sum_{i=1}^{N}\left[\prod_{j=1}^{n} \mu_{F_{j}^{i}}\left(X_{j}\right)\right]}, \quad i=1,2, \ldots, N,
$$

$\phi(X)=\left[\phi_{1}(X), \phi_{2}(X), \ldots, \phi_{N}(X)\right]^{T}$, and $\theta=\left[\theta_{1}, \theta_{2}, \ldots\right.$, $\left.\theta_{N}\right]^{T}$. Hence, the FLS can be rewritten in the following form:

$$
Y(X)=\theta^{T} \phi(X) .
$$

Lemma 6 (see [14]). Let $F(X)$ be a continuous function defined on a compact set $\Omega_{X}$. Then, for any given constant $\epsilon>0$, there exists a FLS $Y(X)$ in the form of (7) such that

$$
\sup _{X \in \Omega_{X}}|F(X)-Y(X)|=\left|F(X)-\theta^{T} \phi(X)\right|<\epsilon .
$$

Similar to [13], the optimal parameter vectors $\theta^{*}$ of FLS are defined as

$$
\theta^{*}=\arg \min _{\theta \in \Omega_{\theta}}\left[\sup _{X \in \Omega_{X}}\left|F(X)-\theta^{T} \phi(X)\right|\right],
$$

where $\Omega_{\theta}$ and $\Omega_{X}$ are compact regions for $\theta$ and $X$, respectively. Furthermore, from Lemma 6 , the fuzzy approximation error $\delta^{*}(X)$ is defined as

$$
F(X)=\theta^{* T} \phi(X)+\delta^{*}(X), \quad \forall X \in \Omega_{X} \subset \mathbb{R}^{n} .
$$

\section{Adaptive Tracking Controller Design and Stability Analysis}

3.1. Adaptive Tracking Controller Design. In this subsection, we shall present an adaptive fuzzy control scheme only based on output variable. Therefore, the high gain observer is introduced to design adaptive output fuzzy tracking controller, and the corresponding lemma is given as follows.

Lemma 7 (see [15]). Consider the following linear system:

$$
\begin{aligned}
& \epsilon_{i} \dot{z}_{i 1}=z_{i 2}, \\
& \epsilon_{i} \dot{z}_{i 2}=-\mu_{i 1} z_{i 2}-z_{i 1}+y_{i}, \quad i=1,2,
\end{aligned}
$$

where $\epsilon_{i}>0$ is a sufficiently small constant and the parameter $\mu_{i 1}$ is appropriately chosen such that $s^{2}+\mu_{i 1} s+1$ is an Hurwitz polynomial. If the output function $y_{i}$ and its $k$ th time derivatives $y_{i}^{(k)}$ are bounded, that is, there exist positive constants $Y_{i 0}, Y_{i k}$ satisfying $\left|y_{i}\right| \leq Y_{i 0},\left|y_{i}^{(k)}\right| \leq Y_{i k}$, then it is obtained that

$$
\eta_{i k}:=\frac{z_{i k}}{\epsilon_{i}^{k-1}}-y_{i}^{k-1}=-\epsilon_{i} \phi_{i}^{(k)}, \quad k=1,2,
$$

where $\phi_{i}:=z_{i 2}+\mu_{i 1} z_{i 1}$ and $\phi_{i}^{(k)}$ represents the $k$ th time derivative of $\phi_{i}$. Moreover, if all the observer states satisfy that $\left|z_{i k}\right| \leq \bar{z}_{i k}$ with $\bar{z}_{i k}>0$, then there exist $\bar{\eta}_{i k}>0$ such that $\left|\eta_{i k}\right| \leq \epsilon_{i} \bar{\eta}_{i k}$.

According to Lemma 6, the estimation of unmeasurable state vector is defined as

$$
\widehat{x}_{i}=\left[x_{i 1}, \frac{z_{i 2}}{\epsilon_{i}}\right]^{T}=\left[\widehat{x}_{i 1}, \widehat{x}_{i 2}\right]^{T}, \quad i=1,2 .
$$

Next, to facilitate control system design from nonaffine form to affine form, the tracking error and the filtered tracking error are defined as $\widehat{e}_{i}=\widehat{x}_{i}-x_{i d}=\left[\widehat{e}_{i 1}, \widehat{e}_{i 2}\right]^{T} \in \mathbb{R}^{2}$ and $\widehat{e}_{i s}=$ $\left[\lambda_{i}, 1\right] \widehat{e}_{i}$, respectively, where $x_{i d}=\left[y_{i d}, \dot{y}_{i d}\right]^{T}$ is the reference state vector and $\lambda_{i}$ is appropriately chosen coefficient such that $s+\lambda_{i}$ is an Hurwitz polynomial; that is, $\widehat{e}_{i} \rightarrow 0$ as $\widehat{e}_{i s} \rightarrow 0$. 
Then, taking the derivative of $\widehat{e}_{i s}$ gives

$$
\dot{\hat{e}}_{i s}=f_{i}\left(x, u_{i}\right)-\ddot{y}_{i d}+\dot{\tilde{x}}_{i 2}+\left[0, \lambda_{i}\right] \widehat{e}_{i}+d_{i},
$$

where $\widehat{x}_{i 2}=x_{i 2}+\tilde{x}_{i 2}$. By using Assumption 3 and the implicit function theorem [16], there exists a unique and continuous ideal control $u_{i}^{*}=U_{i}(x) \in \Omega_{u_{i}}$ such that $f_{i}\left(x, u_{i}^{*}\right)=$ $f_{i}\left(x, U_{i}(x)\right)=0$ for all $x \in \Omega_{x}$, where $\Omega_{x}$ and $\Omega_{u_{i}}$ are two compact sets. By adding and subtracting $\gamma_{i} \widehat{e}_{i s}$ in (14) and applying it to the mean value theorem, we have

$$
\begin{aligned}
\dot{\hat{e}}_{i s}= & f_{i}\left(x, u_{i}^{*}\right)+\frac{\partial f_{i}\left(x, u_{i}^{0}\right)}{\partial u_{i}}\left(u_{i}-u_{i}^{*}\right)+\gamma_{i} \widehat{e}_{i s}-\ddot{y}_{i d} \\
& +\dot{\tilde{x}}_{i 2}+\left[0, \lambda_{i}\right] \widehat{e}_{i}+d_{i}-\gamma_{i} \widehat{e}_{i s} \\
= & \mu_{i} u_{i}-\frac{\partial f_{i}\left(x, u_{i}^{0}\right)}{\partial u_{i}} u_{i}^{*}+\left(\frac{\partial f_{i}\left(x, u_{i}^{0}\right)}{\partial u_{i}}-\mu_{i}\right) u_{i} \\
& +\gamma_{i} e_{i s}+\left[0, \lambda_{i}\right] e_{i}-\ddot{y}_{i d}+\dot{\tilde{x}}_{i 2}+d_{i}+\gamma_{i} \widetilde{e}_{i s} \\
& +\left[0, \lambda_{i}\right] \widetilde{e}_{i}-\gamma_{i} \widehat{e}_{i s},
\end{aligned}
$$

where $u_{i}^{0}$ is some point between zero and $u_{i}, \gamma_{i}>0, \mu_{i}>0$, $\widehat{e}_{i}=e_{i}+\widetilde{e}_{i}$, and $\widehat{e}_{i s}=e_{i s}+\widetilde{e}_{i s}$.

Denote the nonlinear functions $H_{i}\left(x, e_{i}\right)=\left(\partial f_{i}\left(x, u_{i}^{0}\right) /\right.$ $\left.\partial u_{i}\right) u_{i}^{*}-\left(\partial f_{i}\left(x, u_{i}^{0}\right) / \partial u_{i}-\mu_{i}\right) u_{i}-\gamma_{i} e_{i s}-\left[0, \lambda_{i}\right] e_{i}$; it follows from (10) that $H_{i}\left(x, e_{i}\right)$ can be approximated by the following form:

$$
H_{i}\left(x, e_{i}\right)=\theta_{i}^{* T} \phi_{i}\left(x, e_{i}\right)+\delta_{i}^{*}\left(x, e_{i}\right)
$$

for all $\left(x, e_{i}\right) \in \Omega_{x} \times \Omega_{e_{i}}$ with $\Omega_{e_{i}}$ being a compact set. Consequently, substituting (16) into (15) yields

$$
\dot{\hat{e}}_{i s}=\mu_{i} u_{i}-\theta_{i}^{* T} \phi_{i}\left(x, e_{i}\right)+D_{i}(t)-\gamma_{i} \widehat{e}_{i s},
$$

where $D_{i}(t)=-\delta_{i}^{*}\left(x, e_{i}\right)-\ddot{y}_{i d}+\dot{\tilde{x}}_{i 2}+d_{i}+\gamma_{i} \widetilde{e}_{i s}+\left[0, \lambda_{i}\right] \widetilde{e}_{i}$. From Lemma 7 and using Assumptions 2 and 4, it can be concluded that there exists an unknown upper bound $D_{i}^{*}$ such that $\left|D_{i}(t)\right| \leq D_{i}^{*}$.

Remark 8. For the ith nonaffine nonlinear subsystem (3), Assumption 3 plays an important role in the controller design. The reason is that the implicit function theorem is employed to transform the nonaffine nonlinear coupling term into the corresponding affine term based on this assumption. In addition, the similar decoupling method in [13] has been developed; however, it is required that less adjustable parameters are used for the controller design in this paper.

Moreover, the adaptive fuzzy tracking controller is designed for the $i$ th subsystem as follows:

$$
\begin{array}{r}
u_{i}=\mu_{i}^{-1}\left(-\alpha_{i} \widehat{e}_{i s}+\widehat{\theta}_{i}^{T} \phi_{i}\left(\widehat{x}, \widehat{e}_{i}\right)\right. \\
\left.-\frac{\widehat{e}_{i s} \widehat{D}_{i}^{2}}{\widehat{e}_{i s} \widehat{D}_{i} \tanh \left(\widehat{e}_{i s} / \delta_{i}\right)+\delta_{i}}\right)
\end{array}
$$

with adaptation laws

$$
\begin{aligned}
& \dot{\hat{\theta}}_{i}=-\Gamma_{i}\left(\phi_{i}\left(\widehat{x}, \widehat{e}_{i}\right) \widehat{e}_{i s}+\sigma_{i} \widehat{\theta}_{i}\right), \\
& \dot{\widehat{D}}_{i}=-\beta_{i} \sigma_{i} \widehat{D}_{i}+\beta_{i}\left|\widehat{e}_{i s}\right|,
\end{aligned}
$$

where $\widehat{\theta}_{i}$ and $\widehat{D}_{i}$ are the estimate values of $\theta_{i}^{*}$ and $D_{i}^{*}$, respectively, and $\Gamma_{i}=\Gamma_{i}^{T}>0, \alpha_{i}, \delta_{i}, \beta_{i}, \sigma_{i}, i=1,2$, are positive design parameters.

3.2. Stability Analysis. In this subsection, the stability of the resulting closed-loop system is given in the following theorem.

Theorem 9. Consider the MIMO nonaffine nonlinear system (3) with unmeasurable states, under the condition that Assumptions 2-4 hold and the estimation states can be obtained from the high gain observer (11). On the compact set $\Omega_{x} \times \Omega_{e_{i}}$, the adaptive fuzzy tracking controller (18) and the parameter updated laws (19) are constructed; then all the closed-loop system error signals are uniformly ultimately bounded. Moreover, the parameter estimation errors $\widetilde{D}_{i}, \widetilde{\theta}_{i}$ and tracking error $e_{i s}$ remain as the compact sets $\Omega_{\widetilde{D}_{i}}, \Omega_{\widetilde{\theta}_{i}}$, and $\Omega_{e_{i s}}$ in the sense that

$$
\begin{aligned}
& \Omega_{e_{i s}}=\left\{e_{i s} \in \mathbb{R}|| e_{i s} \mid \leq \sqrt{\Omega_{i}}\right\} \\
& \Omega_{\widetilde{D}_{i}}=\left\{\widetilde{D}_{i} \in \mathbb{R}|| \widetilde{D}_{i} \mid \leq \sqrt{\beta_{i} \Omega_{i}}\right\} \\
& \Omega_{\widetilde{\theta}_{i}}=\left\{\widetilde{\theta}_{i} \in \mathbb{R}^{n} \mid\left\|\widetilde{\theta}_{i}\right\| \leq \sqrt{\left.\frac{\Omega_{i}}{\lambda_{\min }\left(\Gamma_{i}^{-1}\right)}\right\}, i=1,2,}\right.
\end{aligned}
$$

$$
\begin{aligned}
\rho_{i 2} & :=\frac{\left(\alpha_{i}+\gamma_{i}\right)^{2} \tau_{i}^{* 2}}{2}+\frac{\tau_{i}^{* 2} \psi_{i}}{2}+2\left\|\theta_{i}^{*}\right\|+\delta_{i}+2 \tau_{i}^{*} D_{i}^{*} \\
& +\frac{\sigma_{i}}{2}\left\|\theta_{i}^{*}\right\|^{2}+\left(2 \kappa \tau_{i}^{*}+\frac{\sigma_{i}}{2}\right) D_{i}^{* 2} .
\end{aligned}
$$

Proof. Define the Lyapunov function $V_{i}\left(e_{i s}, \widetilde{\theta}_{i}, \widetilde{D}_{i}\right)$ as

$$
V_{i}\left(e_{i s}, \widetilde{\theta}_{i}, \widetilde{D}_{i}\right)=\frac{1}{2} e_{i s}^{2}+\frac{1}{2} \beta_{i}^{-1} \widetilde{D}_{i}^{2}+\frac{1}{2} \widetilde{\theta}_{i}^{T} \Gamma_{i}^{-1} \widetilde{\theta}_{i},
$$

where $\widetilde{D}_{i}=\widehat{D}_{i}-D_{i}^{*}$ and $\widetilde{\theta}_{i}=\widehat{\theta}_{i}-\theta_{i}^{*}, i=1,2$, are the parameter estimation errors. Taking the derivative of $V_{i}$ yields

$$
\begin{aligned}
\dot{V}_{i}= & e_{i s} \dot{e}_{i s}+\beta_{i}^{-1} \widetilde{D}_{i} \dot{\widetilde{D}}_{i}+\widetilde{\theta}_{i}^{T} \Gamma_{i}^{-1} \dot{\tilde{\theta}}_{i} \\
= & e_{i s}\left(\mu_{i} u_{i}-\theta_{i}^{* T} \phi_{i}\left(x, e_{i}\right)+D_{i}(t)-\gamma_{i} \widehat{e}_{i s}\right) \\
& +\beta_{i}^{-1} \widetilde{D}_{i} \dot{\widetilde{D}}_{i}+\widetilde{\theta}_{i}^{T} \Gamma_{i}^{-1} \dot{\widetilde{\theta}}_{i} .
\end{aligned}
$$


By substituting (18) into (23), one can obtain that

$$
\begin{aligned}
\dot{V}_{i}= & -\left(\alpha_{i}+\gamma_{i}\right) e_{i s} \widehat{e}_{i s}+\widehat{\theta}_{i}^{T} \phi_{i}\left(\widehat{x}, \widehat{e}_{i}\right) e_{i s} \\
& -\frac{e_{i s} \widehat{e}_{i s} \widehat{D}_{i}^{2}}{\widehat{e}_{i s} \widehat{D}_{i} \tanh \left(\widehat{e}_{i s} / \delta_{i}\right)+\delta_{i}}-\theta_{i}^{* T} \phi_{i}\left(x, e_{i}\right) e_{i s} \\
& +e_{i s} D_{i}+\beta_{i}^{-1} \widetilde{D}_{i} \dot{\tilde{D}}_{i}+\widetilde{\theta}_{i}^{T} \Gamma_{i}^{-1} \dot{\tilde{\theta}}_{i} \\
\leq & -\left(\alpha_{i}+\gamma_{i}\right) e_{i s}^{2}-\left(\alpha_{i}+\gamma_{i}\right) e_{i s} \widetilde{e}_{i s} \\
& +\left(\widehat{\theta}_{i}^{T} \phi_{i}\left(\widehat{x}, \widehat{e}_{i}\right)-\theta_{i}^{* T} \phi_{i}\left(x, e_{i}\right)\right) e_{i s} \\
& -\frac{\widehat{e}_{i s}^{2} \widehat{D}_{i}^{2}}{\widehat{e}_{i s} \widehat{D}_{i} \tanh \left(\widehat{e}_{i s} / \delta_{i}\right)+\delta_{i}} \\
& +\frac{\widetilde{e}_{i s} \widehat{e}_{i s} \widehat{D}_{i}^{2}}{\widehat{e}_{i s} \widehat{D}_{i} \tanh \left(\widehat{e}_{i s} / \delta_{i}\right)+\delta_{i}}+\widehat{e}_{i s} D_{i}-\widetilde{e}_{i s} D_{i} \\
& +\beta_{i}^{-1} \widetilde{D}_{i} \dot{\widetilde{D}}_{i}+\widetilde{\theta}_{i}^{T} \Gamma_{i}^{-1} \dot{\tilde{\theta}}_{i}
\end{aligned}
$$

where $\widetilde{e}_{i s}=\widehat{e}_{i s}-e_{i s}$. Using the adaptive control laws (19), (24) becomes

$$
\begin{aligned}
\dot{V}_{i} \leq & -\left(\alpha_{i}+\gamma_{i}\right) e_{i s}^{2}-\left(\alpha_{i}+\gamma_{i}\right) e_{i s} \widetilde{e}_{i s} \\
& +\left(\widehat{\theta}_{i}^{T} \phi_{i}\left(\widehat{x}, \widehat{e}_{i}\right)-\theta_{i}^{* T} \phi_{i}\left(x, e_{i}\right)\right) e_{i s} \\
& -\frac{\widehat{e}_{i s}^{2} \widehat{D}_{i}^{2}}{\widehat{e}_{i s} \widehat{D}_{i} \tanh \left(\widehat{e}_{i s} / \delta_{i}\right)+\delta_{i}} \\
& +\frac{\widetilde{e}_{i s} \widehat{e}_{i s} \widehat{D}_{i}^{2}}{\widehat{e}_{i s} \widehat{D}_{i} \tanh \left(\widehat{e}_{i s} / \delta_{i}\right)+\delta_{i}}+\left|\widehat{e}_{i s}\right| D_{i}^{*}+\left|\widetilde{e}_{i s}\right| D_{i}^{*} \\
& -\sigma_{i} \widetilde{D}_{i} \widehat{D}_{i}+\left|\widehat{e}_{i s}\right| \widetilde{D}_{i}-\widetilde{\theta}_{i}^{T} \phi_{i}\left(\widehat{x}, \widehat{e}_{i}\right) \widehat{e}_{i s}-\sigma_{i} \widetilde{\theta}_{i}^{T} \widehat{\theta}_{i} .
\end{aligned}
$$

Invoking inequalities $0 \leq b \tanh (b / a) \leq|b|, \forall b \in \mathbb{R}, a>0$ and $|b| \leq b \tanh (b / a)+\kappa a, \forall b \in \mathbb{R}, a>0, \kappa=0.2785$ given in [17], it follows from (25) that

$$
\begin{aligned}
\dot{V}_{i} \leq & -\left(\alpha_{i}+\gamma_{i}\right) e_{i s}^{2}-\left(\alpha_{i}+\gamma_{i}\right) e_{i s} \widetilde{e}_{i s} \\
& +\left(\widehat{\theta}_{i}^{T} \phi_{i}\left(\widehat{x}, \widehat{e}_{i}\right)-\theta_{i}^{* T} \phi_{i}\left(x, e_{i}\right)\right) e_{i s}+\frac{\left|\widehat{e}_{i s}\right| \widehat{D}_{i} \delta_{i}}{\left|\widehat{e}_{i s}\right| \widehat{D}_{i}+\delta_{i}} \\
& +\left|\widetilde{e}_{i s}\right| \widehat{D}_{i}+\kappa\left|\widetilde{e}_{i s}\right| \widehat{D}_{i}^{2}+\left|\widetilde{e}_{i s}\right| D_{i}^{*}-\sigma_{i} \widetilde{D}_{i}^{2} \\
& -\sigma_{i} \widetilde{D}_{i} D_{i}^{*}-\widetilde{\theta}_{i}^{T} \phi_{i}\left(\widehat{x}, \widehat{e}_{i}\right) \widehat{e}_{i s}-\sigma_{i} \widetilde{\theta}_{i}^{T} \widehat{\theta}_{i}
\end{aligned}
$$

For the FLS error term on the right side of (26), adding and subtracting $\theta_{i}^{* T} \phi_{i}\left(\widehat{x}, \widehat{e}_{i}\right)$ yield

$$
\begin{aligned}
\widehat{\theta}_{i}^{T} \phi_{i} & \left(\widehat{x}, \widehat{e}_{i}\right)-\theta_{i}^{* T} \phi_{i}\left(\widehat{x}, \widehat{e}_{i}\right)+\theta_{i}^{* T} \phi_{i}\left(\hat{x}, \widehat{e}_{i}\right) \\
& -\theta_{i}^{* T} \phi_{i}\left(x, e_{i}\right) \\
= & \widetilde{\theta}_{i}^{T} \phi_{i}\left(\hat{x}, \widehat{e}_{i}\right)+\theta_{i}^{* T}\left(\phi_{i}\left(\hat{x}, \widehat{e}_{i}\right)-\phi_{i}\left(x, e_{i}\right)\right) .
\end{aligned}
$$

Using the fact that $\phi_{i}^{T} \phi_{i} \leq 1$ and the triangle inequality, we have

$$
\begin{aligned}
& \widehat{\theta}_{i}^{T} \phi_{i}\left(\widehat{x}, \widehat{e}_{i}\right)-\theta_{i}^{* T} \phi_{i}\left(x, e_{i}\right) \leq \widetilde{\theta}_{i}^{T} \phi\left(\widehat{x}, \widehat{e}_{i}\right)+2\left\|\theta_{i}^{*}\right\| \\
& 2 \widetilde{D}_{i} D_{i}^{*}=\widehat{D}_{i}^{2}-D_{i}^{* 2}-\widetilde{D}_{i}^{2} \geq-D_{i}^{* 2}-\widetilde{D}_{i}^{2} \\
& 2 \widetilde{\theta}_{i}^{T} \widehat{\theta}_{i}=\left\|\widetilde{\theta}_{i}\right\|^{2}+\left\|\widehat{\theta}_{i}\right\|^{2}-\left\|\theta_{i}^{*}\right\|^{2} \geq\left\|\widetilde{\theta}_{i}\right\|^{2}-\left\|\theta_{i}^{*}\right\|^{2} .
\end{aligned}
$$

Invoking (28), (26) becomes

$$
\begin{aligned}
\dot{V}_{i} \leq & -\left(\alpha_{i}+\gamma_{i}\right) e_{i s}^{2}-\left(\alpha_{i}+\gamma_{i}\right) e_{i s} \widetilde{e}_{i s}-\widetilde{\theta}_{i}^{T} \phi_{i}\left(\widehat{x}, \widehat{e}_{i}\right) \widetilde{e}_{i s} \\
& +2\left\|\theta_{i}^{*}\right\|+\frac{\left|\widehat{e}_{i s}\right| \widehat{D}_{i} \delta_{i}}{\left|\widehat{e}_{i s}\right| \widehat{D}_{i}+\delta_{i}}+\left|\widetilde{e}_{i s}\right| \widetilde{D}_{i}+\kappa\left|\widetilde{e}_{i s}\right| \widehat{D}_{i}^{2} \\
& +2\left|\widetilde{e}_{i s}\right| D_{i}^{*}-\frac{\sigma_{i}}{2} \widetilde{D}_{i}^{2}+\frac{\sigma_{i}}{2} D_{i}^{* 2}-\frac{\sigma_{i}}{2}\left\|\widetilde{\theta}_{i}\right\|^{2} \\
& +\frac{\sigma_{i}}{2}\left\|\theta_{i}^{*}\right\|^{2} .
\end{aligned}
$$

From the definition in (12) and the fact that $\widetilde{e}_{i s}=\left[\lambda_{i}, 1\right] \widetilde{e}_{i}$, it can be obtained that $\widetilde{e}_{i s}$ is bounded; that is, there exists a positive constant $\tau_{i}^{*}$ subject to $\left|\widetilde{e}_{i s}\right| \leq \tau_{i}^{*}$. Moreover, by utilizing the inequalities $2 a b \leq(1 / c) a^{2}+c b^{2}, \forall c>0, a, b \in \mathbb{R}$ and $0 \leq a /(a+b)<1, \forall a \geq 0, b>0$, we have

$$
\begin{aligned}
\dot{V}_{i} \leq & -\left(\alpha_{i}+\gamma_{i}\right) e_{i s}^{2}+\frac{1}{2} e_{i s}^{2}+\frac{\left(\alpha_{i}+\gamma_{i}\right)^{2} \tau_{i}^{* 2}}{2}+\frac{1}{2 \psi_{i}}\left\|\widetilde{\theta}_{i}\right\|^{2} \\
& +\frac{\tau_{i}^{* 2} \psi_{i}}{2}+2\left\|\theta_{i}^{*}\right\|+\delta_{i}+\frac{1}{2 \varphi_{i}} \tau_{i}^{* 2}+\frac{\widetilde{D}_{i}^{2} \varphi_{i}}{2}+2 \kappa \tau_{i}^{*} \widetilde{D}_{i}^{2} \\
& +2 \kappa \tau_{i}^{*} D_{i}^{* 2}+2 \tau_{i}^{*} D_{i}^{*}-\frac{\sigma_{i}}{2} \widetilde{D}_{i}^{2}+\frac{\sigma_{i}}{2} D_{i}^{* 2}-\frac{\sigma_{i}}{2}\left\|\widetilde{\theta}_{i}\right\|^{2} \\
& +\frac{\sigma_{i}}{2}\left\|\theta_{i}^{*}\right\|^{2} \leq-\left(\alpha_{i}+\gamma_{i}-\frac{1}{2}\right) e_{i s}^{2}-\frac{1}{2}\left(\sigma_{i}-\frac{1}{\psi_{i}}\right) \\
& \cdot\left\|\widetilde{\theta}_{i}\right\|^{2}-\frac{1}{2}\left(\sigma_{i}-2 \kappa \tau_{i}^{*}\right) \widetilde{D}_{i}^{2}+\left(\frac{\left(\alpha_{i}+\gamma_{i}\right)^{2} \tau_{i}^{* 2}}{2}\right. \\
& +\frac{\tau_{i}^{* 2} \psi_{i}}{2}+2\left\|\theta_{i}^{*}\right\|+\delta_{i}+2 \tau_{i}^{*} D_{i}^{*}+\frac{\sigma_{i}}{2}\left\|\theta_{i}^{*}\right\|^{2} \\
& \left.+\left(2 \kappa \tau_{i}^{*}+\frac{\sigma_{i}}{2}\right) D_{i}^{* 2}\right) \leq-\rho_{i 1} V_{i}+\rho_{i 2},
\end{aligned}
$$

where $\rho_{i 1}$ and $\rho_{i 2}$ are given by the following form:

$$
\begin{aligned}
\rho_{i 1} & :=\min \left\{2\left(\alpha_{i}+\gamma_{i}-\frac{1}{2}\right), \frac{\sigma_{i}-1 / \psi_{i}}{\lambda_{\max }\left(\Gamma_{i}^{-1}\right)},\left(\sigma_{i}-2 \kappa \tau_{i}^{*}\right)\right. \\
& \left.\cdot \beta_{i}\right\} \\
\rho_{i 2}: & :=\frac{\left(\alpha_{i}+\gamma_{i}\right)^{2} \tau_{i}^{* 2}}{2}+\frac{\tau_{i}^{* 2} \psi_{i}}{2}+2\left\|\theta_{i}^{*}\right\|+\delta_{i}+2 \tau_{i}^{*} D_{i}^{*} \\
& +\frac{\sigma_{i}}{2}\left\|\theta_{i}^{*}\right\|^{2}+\left(2 \kappa \tau_{i}^{*}+\frac{\sigma_{i}}{2}\right) D_{i}^{* 2} .
\end{aligned}
$$


By appropriately adjusting the design parameters $\mu_{i}, \alpha_{i}$, $\beta_{i}, \gamma_{i}, \sigma_{i}, \delta_{i}$, and $\psi_{i}$ such that $\alpha_{i}+\gamma_{i}-1 / 2>0, \sigma_{i}-1 / \psi_{i}>0$, and $\sigma_{i}-2 \kappa \tau_{i}^{*}>0$, respectively, the closed-loop system stability can be guaranteed. Finally, by multiplying $e^{\rho_{i 1} t}$ and integrating over $[0, t]$ on both sides of $(30)$, we have

$$
V_{i} \leq\left(\left.V_{i}\right|_{t=0}-\frac{\rho_{i 2}}{\rho_{i 1}}\right) e^{-\rho_{i 1} t}+\frac{\rho_{i 2}}{\rho_{i 1}} \leq\left. V_{i}\right|_{t=0}+\frac{\rho_{i 2}}{\rho_{i 1}} .
$$

It follows from (22) and (32) that

$$
\begin{aligned}
& \left|e_{i s}\right| \leq \sqrt{2\left(\left.V_{i}\right|_{t=0}+\frac{\rho_{i 2}}{\rho_{i 1}}\right)} \\
& \left|\widetilde{D}_{i}\right| \leq \sqrt{2 \beta_{i}\left(\left.V_{i}\right|_{t=0}+\frac{\rho_{i 2}}{\rho_{i 1}}\right)} \\
& \left\|\widetilde{\theta}_{i}\right\| \leq \sqrt{\frac{2\left(\left.V_{i}\right|_{t=0}+\rho_{i 2} / \rho_{i 1}\right)}{\lambda_{\text {min }}\left(\Gamma_{i}^{-1}\right)} .} .
\end{aligned}
$$

Consequently, the parameter estimation errors $\widetilde{D}_{i}, \widetilde{\theta}_{i}$ and tracking error $e_{s}$ are bounded from (31) and remain as the compact sets $\Omega_{\widetilde{D}_{i}}, \Omega_{\widetilde{\theta}_{i}}$, and $\Omega_{e_{i s}}$ in the sense that

$$
\begin{aligned}
& \Omega_{e_{i s}}=\left\{e_{i s} \in \mathbb{R}|| e_{i s} \mid \leq \sqrt{\Omega_{i}}\right\} \\
& \Omega_{\widetilde{D}_{i}}=\left\{\widetilde{D}_{i} \in \mathbb{R}|| \widetilde{D}_{i} \mid \leq \sqrt{\beta_{i} \Omega_{i}}\right\} \\
& \Omega_{\widetilde{\theta}_{i}}=\left\{\widetilde{\theta}_{i} \in \mathbb{R}^{n} \mid\left\|\widetilde{\theta}_{i}\right\| \leq \sqrt{\frac{\Omega_{i}}{\lambda_{\text {min }}\left(\Gamma_{i}^{-1}\right)}}\right\}, \quad i=1,2,
\end{aligned}
$$

where $\Omega_{i}=2\left(\left.V_{i}\right|_{t=0}+\rho_{i 2} / \rho_{i 1}\right)$ with $\rho_{i 1}$ and $\rho_{i 2}$ defined as (31). The proof is completed.

Remark 10. It is worth mentioning that the authors in [13] considered the adaptive fuzzy tracking control problem for a class of SISO nonaffine nonlinear systems. However, the approach proposed in [13] cannot be applied to MIMO nonaffine nonlinear casting systems with immeasurable coupling states. In this paper, the mean value theorem and the fuzzy approximation method are employed to transform the nonaffine nonlinear systems to the corresponding affine nonlinear systems. Also, based on Lyapunov stability analysis, it is shown that the proposed adaptive fuzzy output tracking control scheme can guarantee that the roll gap $x_{g}$ and the molten steel level $x_{h}$ can track to the empirical reference signals.

\section{Simulations and Experimental Analysis}

In this section, in order to verify the effectiveness of the proposed adaptive fuzzy control method, the following numerical simulation is performed for MIMO nonaffine nonlinear system (3), and the corresponding system parameters are selected as $R=150 \mathrm{~mm}, L=200 \mathrm{~mm}, M=300 \mathrm{~kg}$, and $v_{r}=10 \mathrm{mpm}$. These values are chosen from [9]. In addition, the initial roll gap and the desired roll gap are set to be $0 \mathrm{~mm}$ and $3 \mathrm{~mm}$, and the initial molten steel level and desired molten steel level are set to be $20 \mathrm{~mm}$ and $70 \mathrm{~mm}$, respectively.

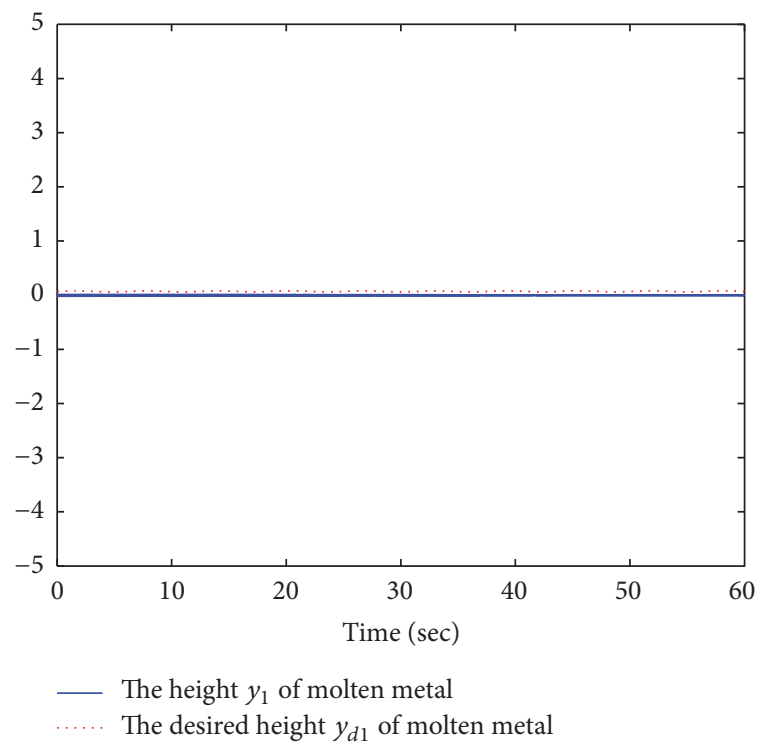

Figure 2: Trajectories of the system output $y_{1}$ and the desired reference signal $y_{d 1}$.

Furthermore, the fuzzy membership functions are chosen as follows:

$$
\begin{aligned}
& \mu_{F_{j}^{1}}=\exp \left[-\frac{\left(X_{j}+1.5\right)^{2}}{2}\right], \\
& \mu_{F_{j}^{2}}=\exp \left[-\frac{\left(X_{j}+1\right)^{2}}{2}\right], \\
& \mu_{F_{j}^{3}}=\exp \left[-\frac{\left(X_{j}+0.5\right)^{2}}{2}\right], \\
& \mu_{F_{j}^{4}}=\exp \left[-\frac{\left.X_{j}^{2}\right],}{2}\right], \\
& \mu_{F_{j}^{5}}=\exp \left[-\frac{\left(X_{j}-0.5\right)^{2}}{2}\right], \\
& \mu_{F_{j}^{6}}=\exp \left[-\frac{\left(X_{j}-1\right)^{2}}{2}\right], \\
& \mu_{F_{j}^{7}}=\exp \left[-\frac{\left(X_{j}-1.5\right)^{2}}{2}\right],
\end{aligned}
$$

Define fuzzy basis functions as

$$
\phi_{i}(X)=\frac{\prod_{j=1}^{n} \mu_{F_{j}^{i}}\left(X_{j}\right)}{\sum_{i=1}^{7}\left[\prod_{j=1}^{n} \mu_{F_{j}^{i}}\left(X_{j}\right)\right]}, \quad i=1,2, \ldots, 7,
$$

where $X=\left[X_{1}, X_{2}, \ldots, X_{n}\right]^{T}$. 


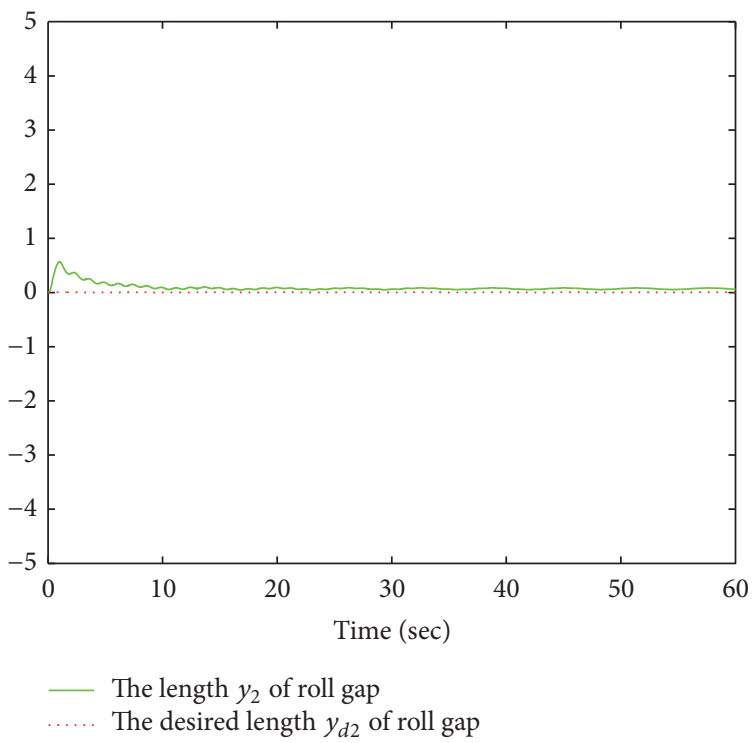

FIGURE 3: Trajectories of the system output $y_{2}$ and the desired reference signal $y_{d 2}$.

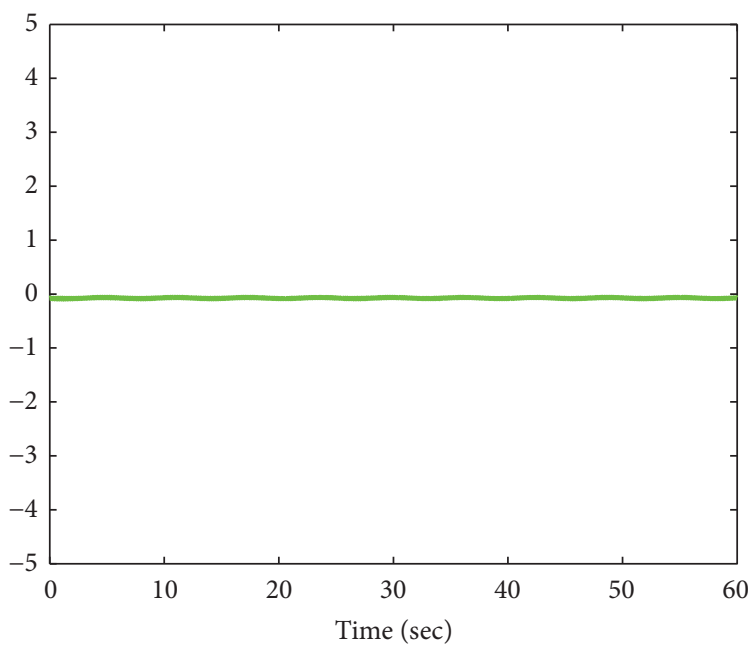

The tracking error of molten steel level

FIGURE 4: Trajectory of the system tracking error of molten steel level.

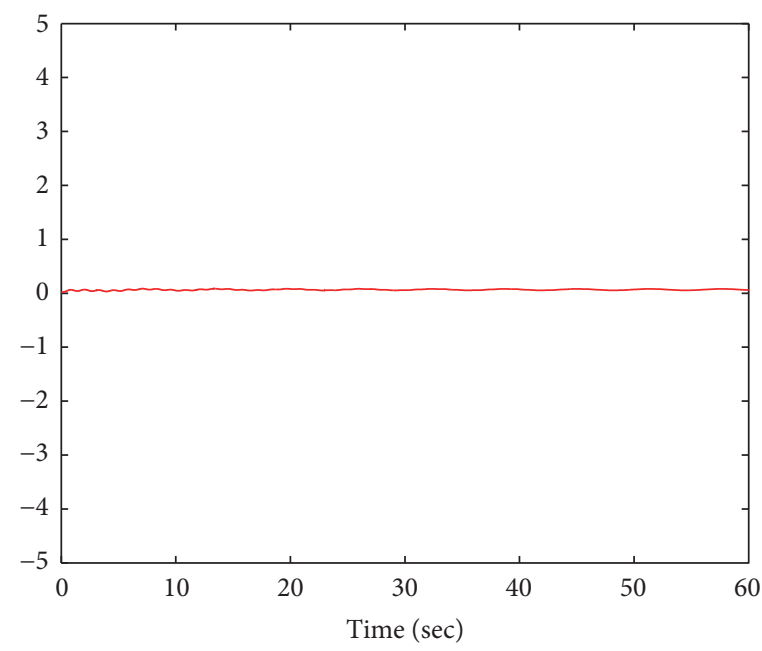

The tracking error of roll gap

FIGURE 5: Trajectory of the system tracking error of roll gap. 


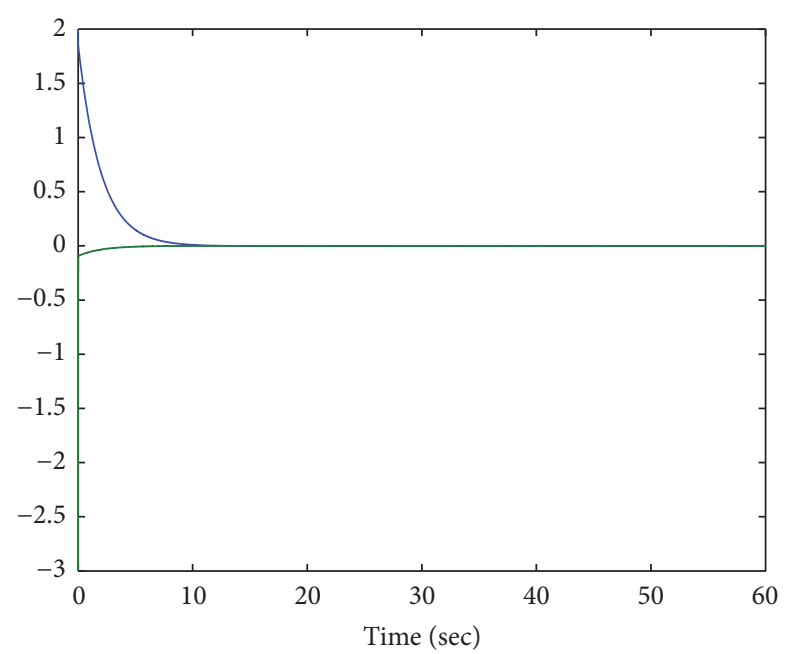

- The observer state $\widehat{x}_{11}$

— The observer state $\hat{x}_{12}$

Figure 6: The observer states $\widehat{x}_{11}$ and $\widehat{x}_{12}$.

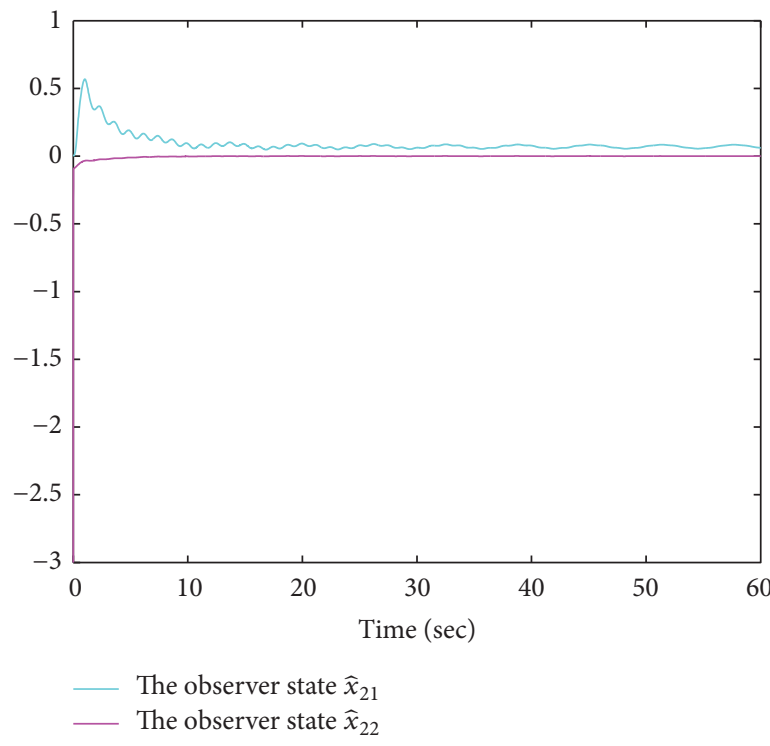

FIGURE 7: The observer states $\widehat{x}_{21}$ and $\hat{x}_{22}$.

Besides, the simulation parameters are chosen as $\mu_{i}=5$, $\alpha_{i}=5, \beta_{i}=0.2, \Gamma_{i}=10, \sigma_{i}=10$, and $\delta_{i}=0.1, i=$ 1,2 , and the initial values are selected as $x_{1}(0)=[0.02,0]^{T}$, $x_{2}(0)=[0,0.1]^{T}, \widehat{x}_{1}(0)=[2,-3]^{T}, \widehat{x}_{2}(0)=[0,-1]^{T}, \widehat{\theta}_{1}(0)=$ $\widehat{\theta}_{2}(0)=[0,0.1,0,0.1,0,0,0.1]^{T}$, and $\widehat{D}_{1}(0)=\widehat{D}_{2}(0)=5$. The simulation results are obtained in Figures 2-11. From Figures 2 and 3 , the system output signals of $y_{i}$ and the reference signals $y_{d i}$, respectively, can be seen. Figures 4 and 5 show that the tracking performance is satisfactory and the output tracking errors of the roll gap and the molten steel level can converge to the desired neighborhoods of the origin. The curves of the estimations states are given in Figures 6 and 7. The boundedness of parameter estimations $\widehat{\theta}_{i}$ and $\widehat{D}_{i}$, as well as the designed control $u_{i}$, is demonstrated in Figures 8-11. Besides, the casting 7075 aluminum alloy strip is shown in Figure 12. It can also be seen that the casting strip is flat and there are no obvious cracks on the surface.

\section{Conclusion}

This paper studies the robust adaptive fuzzy tracking control problem for a class of twin-roll strip casting systems. Based on fuzzy logic systems (FLSs) to approximate the compounded nonlinear functions, a novel adaptive fuzzy output tracking control scheme is developed by using the high gain observer. The mean value theorem is employed to decouple the nonaffine nonlinear systems, and thus it is proved that all 


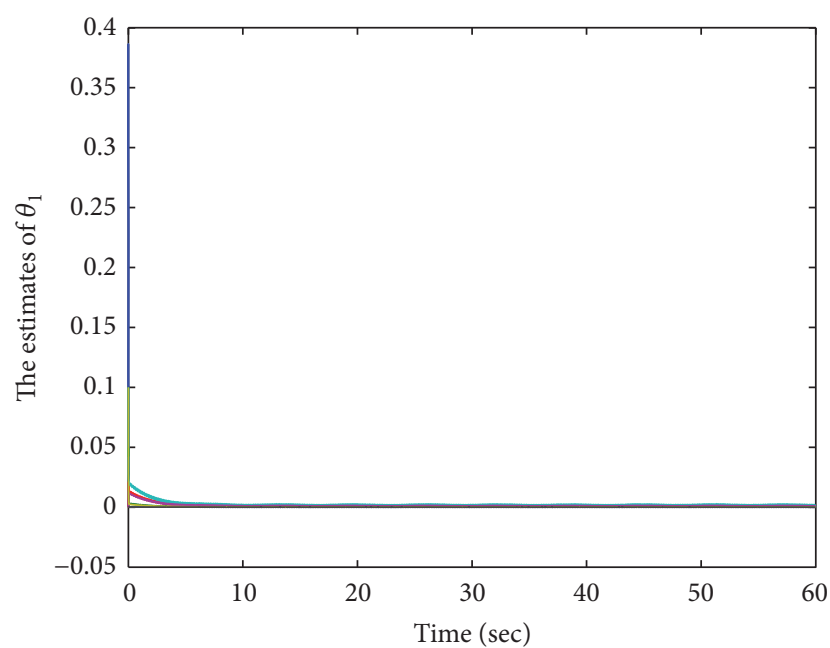

$\begin{array}{ll}\text { The estimate of } \theta_{11} & - \text { The estimate of } \theta_{15} \\ \text { The estimate of } \theta_{12} & \text { The estimate of } \theta_{16} \\ \text { The estimate of } \theta_{13} & - \text { The estimate of } \theta_{17}\end{array}$

The estimate of $\theta_{14}$

FIGURE 8: The response curves of $\widehat{\theta}_{1}$.

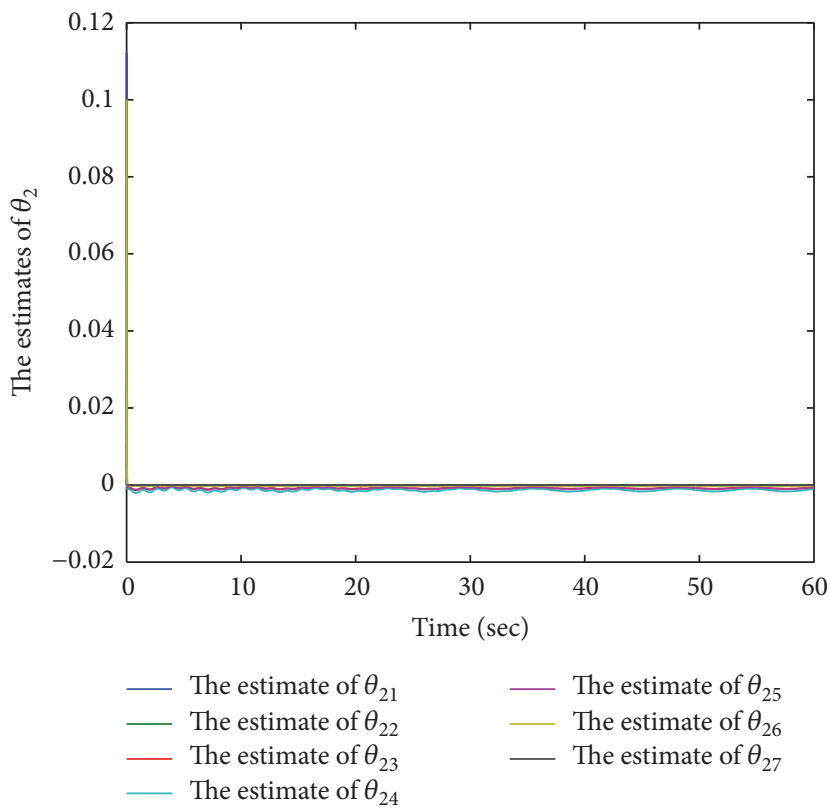

Figure 9: The response curves of $\widehat{\theta}_{2}$.

the closed-loop signals are bounded and the systems output tracking errors of the roll gap and the molten steel level can converge to the desired neighborhoods of the origin via the Lyapunov stability analysis. Simulation results show the effectiveness of the proposed adaptive control approach.

\section{Conflicts of Interest}

The authors declare that they have no conflicts of interest.

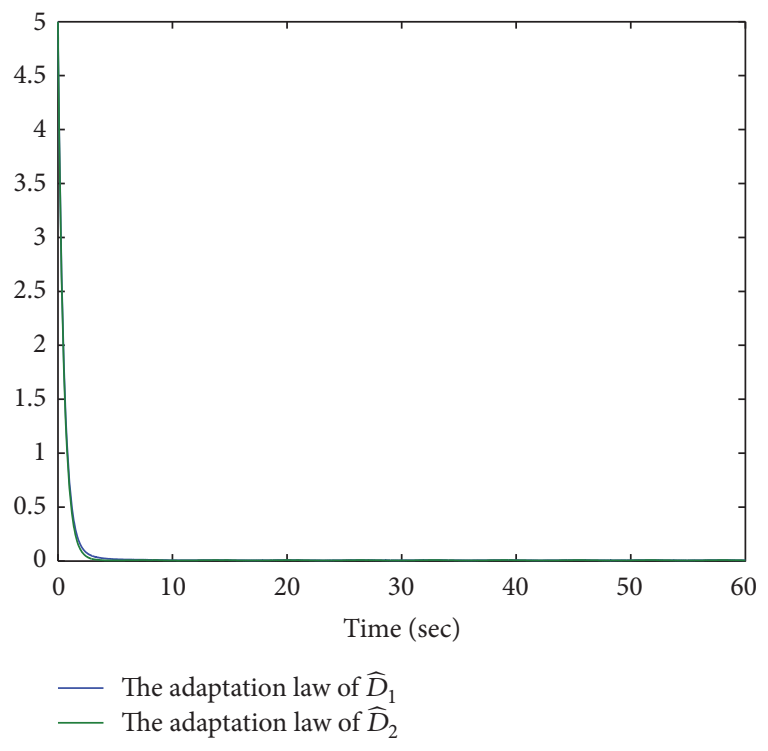

FIGURE 10: The response curves of the adaptive laws.

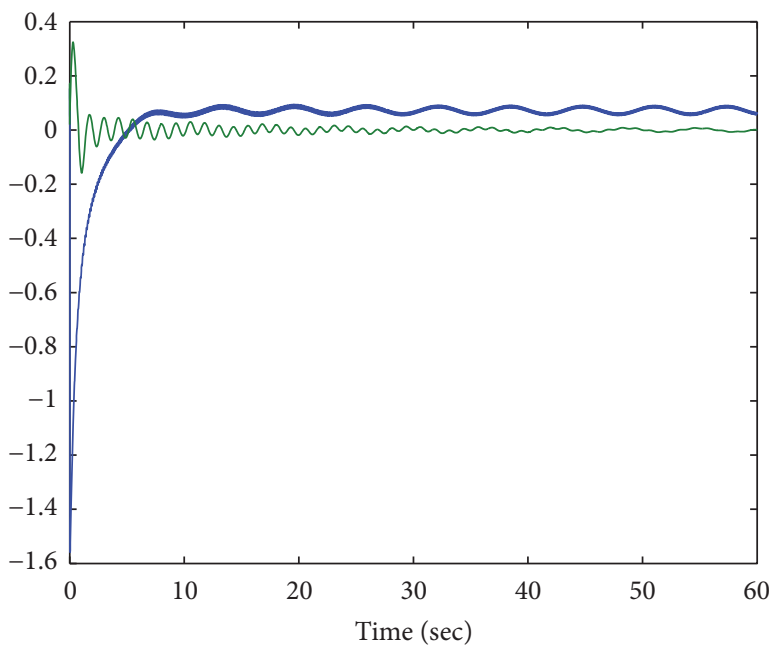

- The control input $u_{1}$

- The control input $u_{2}$

FIGURE 11: The respond curves of the control signals.

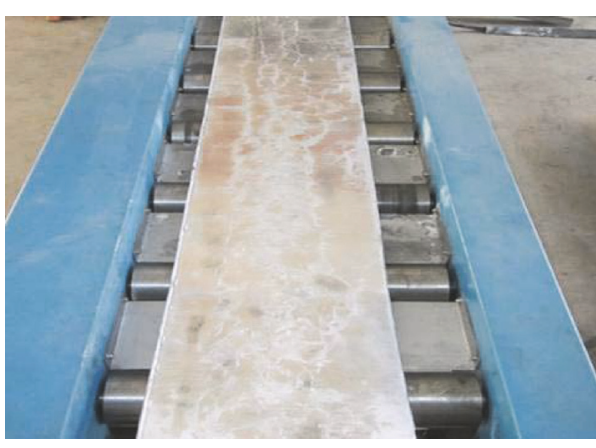

FIGURE 12: The casting 7075 aluminum alloy strip image showing the smooth surface in twin-roll strip process. 


\section{Acknowledgments}

This work was supported in part by the Fundamental Research Funds of Anshan Municipal Government.

\section{References}

[1] R. Cook, P. G. Grocock, P. M. Thomas, D. V. Edmonds, and J. D. Hunt, "Development of the twin-roll casting process," Journal of Materials Processing Technology, vol. 55, no. 2, pp. 76-84, 1995.

[2] D.-Y. Ju, H.-Y. Zhao, X.-D. Hu, K. Ohori, and M. Tougo, "Thermal flow simulation on twin roll casting process for thin strip production of magnesium alloy," Materials Science Forum, vol. 488-489, pp. 439-444, 2005.

[3] S. F. Graebe, "Control design and implementation in continuous steel casting," IEEE Control Systems, vol. 15, no. 4, pp. 64-71, 1995.

[4] T. Hesketh, D. J. Clements, and R. Williams, "Adaptive mould level control for continuous steel slab casting," Automatica, vol. 29, no. 4, pp. 851-864, 1993.

[5] K.-S. Hong, J.-G. Kim, and M. Tomizuka, "Control of strip casting process: decentralization and optimal roll force control," Control Engineering Practice, vol. 9, no. 9, pp. 933-945, 2001.

[6] Y. Park and H. Cho, "A fuzzy logic controller for the molten steel level control of strip casting processes," Control Engineering Practice, vol. 13, no. 7, pp. 821-834, 2005.

[7] H.-Y. Chen and S.-J. Huang, "Self-organizing fuzzy controller for the molten steel level control of a twin-roll strip casting process," Journal of Intelligent Manufacturing, vol. 22, no. 4, pp. 619-626, 2011.

[8] H.-Y. Chen and S.-J. Huang, "Adaptive neural network controller for the molten steel level control of strip casting processes," Journal of Mechanical Science and Technology, vol. 24, no. 3, pp. 755-760, 2010.

[9] W. Zhang, D. Ju, H. Zhao, X. Hu, Y. Yao, and Y. Zhang, "A decoupling control model on perturbation method for twin-roll casting magnesium alloy sheet," Journal of Materials Science and Technology, vol. 31, no. 5, pp. 517-522, 2015.

[10] H. Wang, L. Zhou, Y. Zhang, Y. Cai, and J. Zhang, "Effects of twin-roll casting process parameters on the microstructure and sheet metal forming behavior of 7050 aluminum alloy," Journal of Materials Processing Technology, vol. 233, pp. 186-191, 2016.

[11] Z. Zhang, S. Xu, and B. Zhang, "Asymptotic tracking control of uncertain nonlinear systems with unknown actuator nonlinearity," IEEE Transactions on Automatic Control, vol. 59, no. 5, pp. 1336-1341, 2014.

[12] Z. Zhang, S. Xu, and B. Zhang, "Exact tracking control of nonlinear systems with time delays and dead-zone input," Automatica, vol. 52, pp. 272-276, 2015.

[13] L.-B. Wu and G.-H. Yang, "Adaptive fuzzy tracking control for a class of uncertain nonaffine nonlinear systems with dead-zone inputs," Fuzzy Sets and Systems, vol. 290, pp. 1-21, 2016.

[14] L. X. Wang, Adaptive Fuzzy Systems and Control, Prentice-Hall, Englewood Cliffs, NJ, USA, 1994.

[15] M. Chen and S. S. Ge, "Direct adaptive neural control for a class of uncertain nonaffine nonlinear systems based on disturbance observer," IEEE Transactions on Cybernetics, vol. 43, no. 4, pp. 1213-1225, 2013.

[16] R. G. Bartle, The Elements of Real Analysis, Wiley, Hoboken, NJ, USA, 1964.
[17] M. M. Polycarpou, "Stable adaptive neural control scheme for nonlinear systems," IEEE Transactions on Automatic Control, vol. 41, no. 3, pp. 447-451, 1996. 


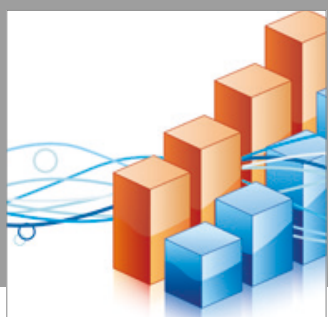

Advances in

Operations Research

vatersals

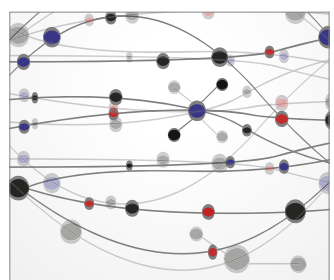

\section{The Scientific} World Journal
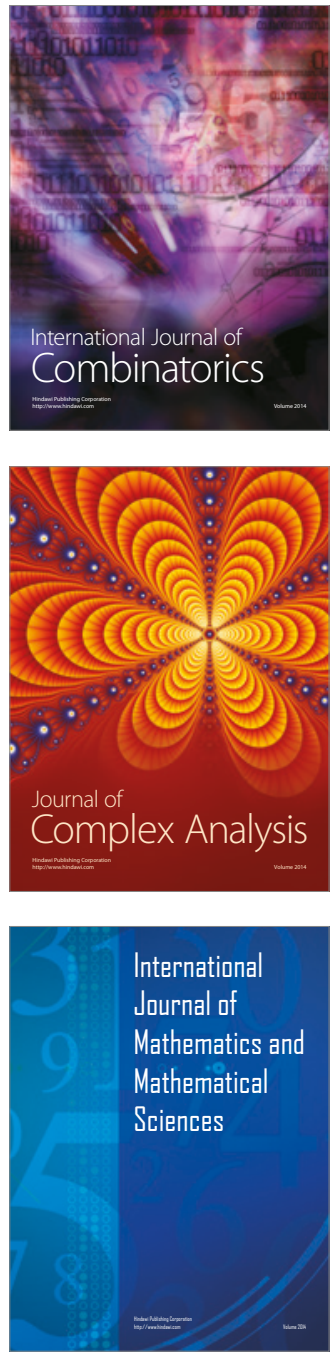
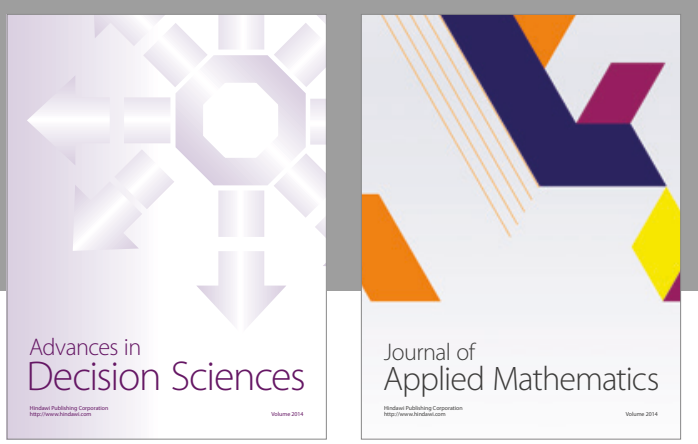

Algebra

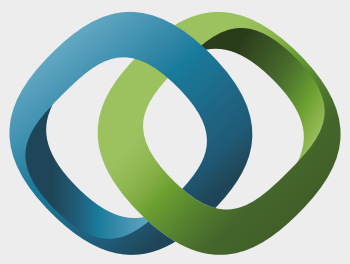

\section{Hindawi}

Submit your manuscripts at

https://www.hindawi.com
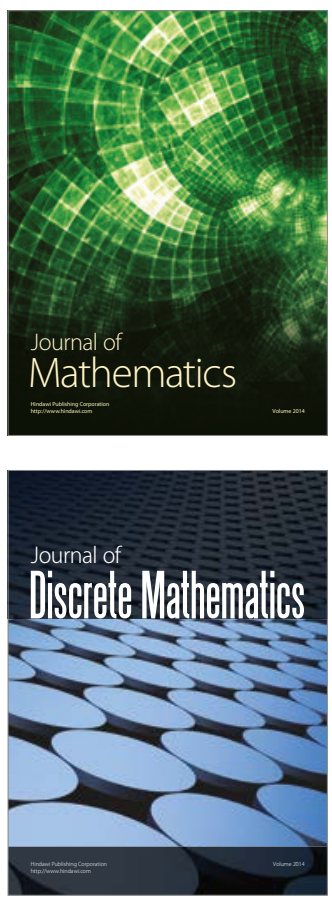

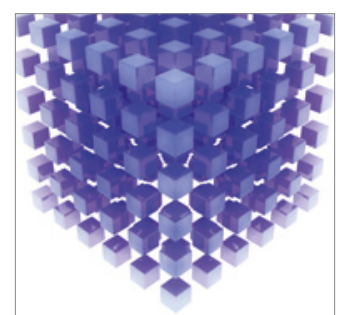

Mathematical Problems in Engineering
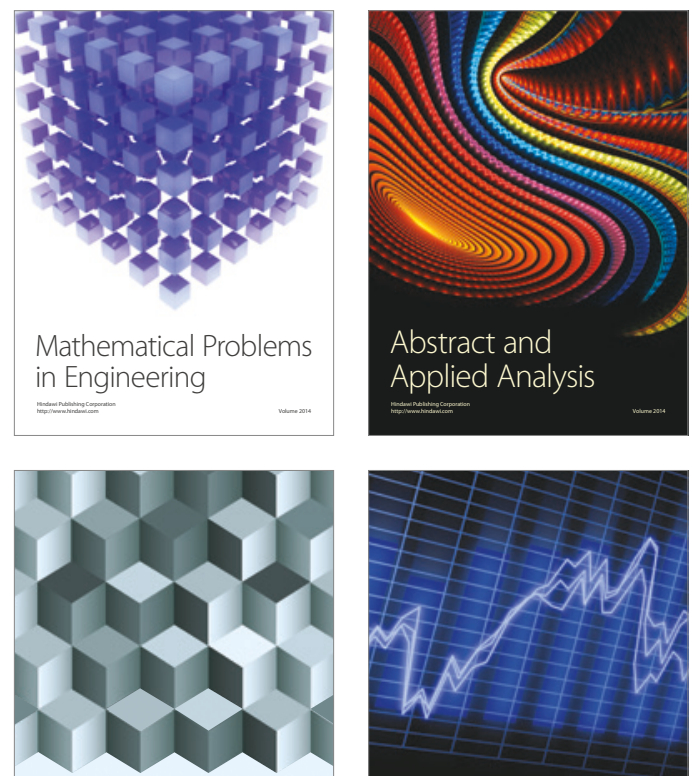

Journal of

Function Spaces

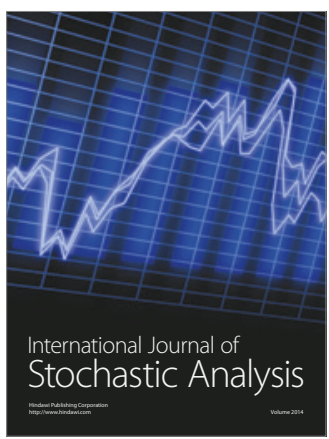

Probability and Statistics
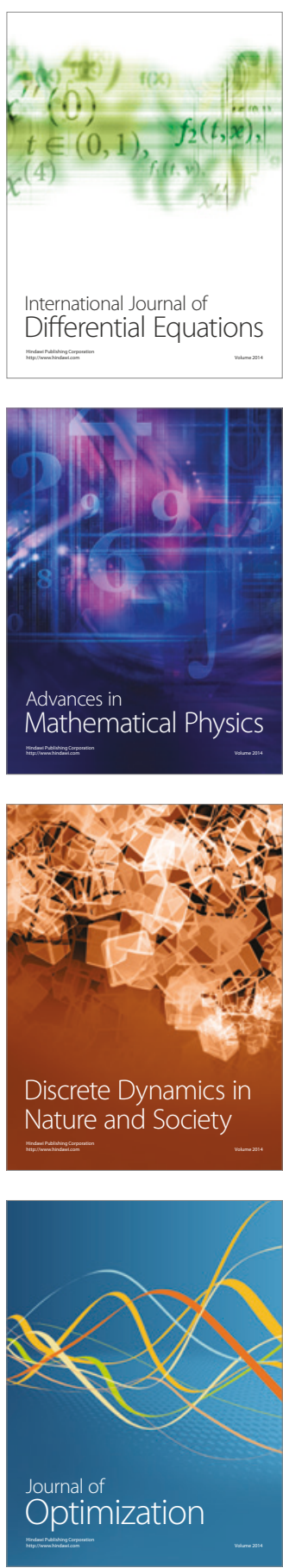